\title{
Contusões em carcaças bovinas: Fatores de ocorrência e influências do bem-estar animal na cadeia produtiva: revisão
}

\author{
[Bovine carcasses contusions: Factors of occurrence and influences on animal welfare and the \\ productive chain - a review]
}

\section{"Revisão/Review"}

\author{
Javier Alexander Bethancourt Garcia*, Tiago Albandes Fernandes, Ricardo Zambarda Vaz
}

Departamento de Zootecnia, Universidade Federal de Pelotas, Pelotas-RS, Brasil.

*Autor para correspondência/Corresponding author: E-mail: javierbethancourt_@hotmail.com

\begin{abstract}
Resumo
A cadeia produtiva da bovinocultura brasileira vem apresentando um constante crescimento no PIB do agronegócio nos últimos três anos, devido à modernização e organização da cadeia, sustentada pela introdução de novas tecnologias, ao avanço genético, ao manejo nutricional, bem como às pesquisas realizadas em diferentes instituições do País. Entretanto, ainda persistem os problemas de bem-estar animal, os quais são evidenciados nos frigoríficos, através do aumento de lesões nas carcaças bovinas, demostrando um reflexo negativo para a bovinocultura. Desta maneira, a presente revisão objetivou reunir e discutir informações sobre as contusões em carcaças bovinas, suas características e seus prejuízos na cadeia produtiva. Estas lesões são observadas durante a esfola na linha de abate e estão relacionadas aos erros de manejo e ao sofrimento dos animais. Devido à essa situação, a cadeia produtiva sofre um impacto direto na lucratividade. Desta forma, ocorre a necessidade da implementação de novas estratégias, aperfeiçoando os manejos utilizados atualmente, como a inclusão de programas de capacitação para a equipe de trabalho em todo o sistema produtivo, a adoção de um planejamento logístico na hora de realizar o transporte dos animais aos abatedouros frigoríficos, bem como o incremento de fiscalizações para garantir o cumprimento das leis. A correção destes fatores reduzirá a presença de contusões, proporcionando a obtenção de um produto de melhor qualidade.
\end{abstract}

Palavras-chave: abatedouros frigoríficos; bovinos de corte; carne; manejo pré-abate; transporte.

\begin{abstract}
The Brazilian cattle breeding production chain has been showing a constant growth in the agribusiness GDP in the last three years due to the modernization and organization of the chain, supported by the introduction of new technologies, genetic advancement, nutritional management, as well as research carried out in different institutions of the country. However, animal welfare problems persist, which are evidenced in the slaughterhouses, through the increase of lesions in cattle carcasses, which reflects poorly on beef cattle farming. In this way, the present review aimed to gather and discuss information about the cattle carcass bruises, their characteristics, and their losses in the production chain. These lesions are observed during skinning on the slaughter line and are related to management errors and the suffering of the animals. Due to this situation, the productive chain has a direct impact on profitability. In this way, it is necessary to implement new strategies, improving the management currently used, such as the inclusion of training programs for the work team throughout the production system, the adoption of a logistics planning for the animal transport to the slaughterhouses, as well as the increase of inspections to ensure compliance with the law. The correction of these factors will reduce presence of bruises, providing a better quality product.
\end{abstract}

Keyword: beef cattle; meat; pre-slaughter management; slaughterhouse; transport.

\section{Introdução}

Ao longo dos últimos vinte anos cerca de $80 \%$ do mercado consumidor, principalmente de países mais desenvolvidos e industrializados, têm

se tornado mais exigente e preocupado com a segurança, qualidade da carne consumida e o bemestar dos animais (Mitchell, 2001; Pereira de Abreu 
et al., 2012). Dessa forma, de acordo com Gallo e Néstor (2008), cuidados têm sido implantados no manejo dos animais, os quais visam a obtenção de carcaças em conformidade com o bem-estar animal (ausência ou minimização de contusões).

Petroni et al. (2013) salientam que a ausência de um programa de bem-estar animal acarreta desprestígio no mercado internacional de carnes, causando enormes perdas diretas e indiretas para a cadeia produtiva da carne bovina, devido à remoção de áreas acometidas por lesões oriundas do mau manejo. Além disso, a presença de hematomas nas diferentes regiões da carcaça, está relacionado ao sofrimento animal, sendo, nos dias de hoje, muito estudada a relação entre bem-estar animal e a qualidade do produto final. Por esta razão, práticas adequadas durante as etapas do préabate reduzem significativamente a incidência de carcaças lesadas no abatedouro frigorífico (Paranhos da Costa., 2012).

Neste sentido, a presente revisão objetivou reunir e discutir informações sobre as contusões em carcaças bovinas, suas características e seus prejuízos na cadeia produtiva.

\section{Desenvolvimento}

Fatores que aumentam o risco de hematomas nas carcaças bovinas:

O hematoma pode ser definido como uma lesão onde os tecidos foram esmagados, com ruptura no fornecimento vascular e acumulação de sangue e soro, mas sem descontinuidade da pele (Mendonça et al., 2018). De acordo com Chambers et al. (2001), as contusões são originadas por golpe ou impacto físico de pedras, chifres, objetos de metal ou de madeira, produto da deterioração dos currais de manejo, entre outros, podendo ocorrer em qualquer etapa do manejo pré-abate. Entretanto, para solucionar os problemas de bem-estar animal é importante conhecer os fatores causais e estabelecer práticas que possam diminuir os riscos, que ocasionam perdas na cadeia produtiva da carne bovina.

\section{Manejo pré-abate de bovinos:}

O pré-abate engloba etapas fundamentais, tais como o embarque, o transporte, o desembarque e as instalações de pré-abate e de abate, sendo estas, até o momento, desconhecidas para os animais (Duarte et al., 2014), tendo os mesmos, reações adversas aos manejos e instalações (Fernandes et al., 2017a). Por essa razão, acredita-se que para garantir uma carne de qualidade é necessário um estudo minucioso, a fim de detectar pontos críticos e estabelecer um programa de qualidade de serviços no manejo com os bovinos (Miranda-De La Lama et al., 2012).

Um sistema de produção animal que não é acompanhado por boas práticas de manejo, geralmente eleva a ocorrência de contusões com impactos econômicos negativos na cadeia da carne (Huertas et al., 2015). Embora a manipulação dos bovinos no manejo pré-abate seja inevitavelmente estressante, é aconselhável manter esse estresse em níveis mínimos (Gallo e Néstor, 2008).

As operações realizadas no abatedouro frigorífico também influenciam nos parâmetros fisiológicos e comportamentais do animal, pois irregularidades na manutenção de equipamentos e instalações, uso de mão de obra não qualificada e supervisão ineficiente provocam falhas nos processos de acomodação, condução, atordoamento e sangria. Estes efeitos causam irregularidades como o incremento nos valores do pH da carne, situação que tende a produzir carnes mais escuras, firmes e secas, anomalia denominada "Dark-cutting beef" (carne bovina de corte escuro), que apresenta como consequência uma redução radical no tempo de vida útil do produto (Ludtke et al., 2012; Mendonça et al., 2017).

\section{Alterações climáticas:}

A incidência de contusões pode ser atribuída às alterações climáticas que ocorrem nas estações do ano, como a radiação solar intensa, umidade relativa, temperatura, velocidade do vento e precipitação, fatores que podem provocar alterações em respostas fisiológicas e de comportamento dos animais criados nos diferentes sistemas de produção.

Os sistemas de produção extensivos apresentam aspectos favoráveis para o bem-estar animal, especificamente quando se refere a expressão de comportamento natural (Paranhos da Costa et al., 2012). Entretanto, quando as temperaturas são extremas (calor o frio) e não se mantem um manejo estratégico nutricional adequado, surgem diversos prejuízos nos indicadores produtivos como menores ganhos de peso, extensão do período de engorda, redução da conversão alimentar e redução da ingestão alimentar, originados pela escassez da oferta forrageira ou baixo valor nutricional, o que aumenta a dificuldade dos animais frente à necessidade de conseguir alimento. Esta situação seria suficiente para incrementar os níveis de 
estrese e o risco de contusões. Nesse sentido, Gallo e Néstor (2008) manifestam que manter uma dieta com pastagens de boa qualidade, que permita reservas adequadas de glicogênio muscular, ajuda a manter reservas de energia suficientes para enfrentar de melhor forma as situações estressantes.

No entanto, nos sistemas de produção intensivos, onde a supervisão do homem é mais frequente, se empregam diversas alternativas para diminuir o impacto das alterações climáticas, como o fornecimento de forragem e concentrado de boa qualidade, além da utilização de nebulizadores associados a ventiladores e instalações do sombreamento, gerando melhoras significativas na produção e no bem-estar dos animais (Tucker et al., 2008).

\section{Transporte:}

$\mathrm{O}$ transporte, incluindo o embarque e o desembarque dos animais, são fases críticas para a obtenção de carne de qualidade. Quanto menor for o estresse sofrido pelos animais neste período, associado a um tempo de descanso adequado nos currais do abatedouro frigorífico, melhor será a qualidade das carcaças e a aceitação do mercado, principalmente externo, o qual está dando preferência por abatedouros frigoríficos que empregam técnicas humanitárias. Assim, tanto do ponto de vista do bem-estar quanto do ponto de vista econômico, é importante controlar e minimizar fatores indutores de estresse durante o transporte para o abate (Bulitta et al., 2015).

No Brasil, anualmente são abatidos mais de 40 milhões de bovinos (Anualpec, 2017), sendo a maior parte desses animais transportados por rodovias, se utilizando caminhões de diversas capacidades, variando de 18 a 48 animais, com diversos compartimentos, onde, muitas vezes, os animais podem estar distribuídos em dois pisos com diferentes densidades (Bertoloni et al., 2012).

\section{Distância percorrida:}

Fatores como o tempo e a distância de transporte se mostram positivamente correlacionados com a ocorrência de contusões em bovinos (Mendonça et al., 2016). Quando se avalia a distância, se deve levar em consideração as variações nas condições existentes, como topografia do terreno, qualidade das estradas, fatores climáticos, bem como particularidades da fazenda de origem, a qual poderia contar com muitas porteiras, e provocaria um incremento no número de freadas e arranques dos caminhões, o que pode ocasionar a perda de equilíbrio dos animais (Bethancourt-Garcia et al., 2019).

O longo tempo de viagem demostra ser um fator muito estressante, devido à privação de alimento e água, o que estimula a degradação das reservas de gordura, prejudicando o bem-estar dos animais. Tais fatos provocam respostas neuroendócrinas e de fase aguda, as quais reduzem o desempenho dos bovinos (Marques et al., 2012). Não obstante, quando o transporte de longas distâncias é realizado com condições adequadas, incluindo baixa densidade de carga e tempo de permanência suficiente com provisão de alimentos e água, pode não causar estresse severo para os animais (Ishiwata et al., 2008).

A rede de rodovias no Brasil tem mais de 1,6 milhões de quilômetros, sendo 1,3 milhões de rodovias municipais, $230 \mathrm{mil}$ de rodovias estaduais e 73 mil de rodovias federais, das quais somente $27,1 \%$ das estradas estão classificadas como ótimas ou boas e 72,9\% apresentam algum tipo de deficiência, estando classificadas como regulares, ruins ou péssimas, o que justifica o incremento de contusões por estradas em condições precárias (CNT, 2016). Esta situação pode ser observada em estudo realizado por Andrade et al. (2008), que avaliando a influência do transporte rodoviário sobre a ocorrência de lesões em 121 bovinos, observaram que $84,3 \%$ dos animais transportados tiveram uma ou mais lesões, totalizando 270 lesões, resultando na remoção de 0,5 e $0,6 \mathrm{Kg}$ de carne/animal, evidenciando um maior número de lesões em bovinos transportados por mais de três horas e distâncias maiores de $52 \mathrm{~km}$ por estradas não pavimentadas.

\section{Densidade de carga:}

A definição da densidade de carga correta é fundamental para garantir melhores condições de bem-estar para os animais durante o transporte. Entretanto, ainda é comum definir a densidade de carga de bovinos sem considerar aspectos necessários como a raça, peso, presença de chifre e sexo dos animais a serem transportados. Isso acontece, possivelmente, pelas pressões comerciais existentes, as quais, consequentemente, geram um aumento nas densidades de carga, com a finalidade de reduzir os custos gerados pelo transporte. Entretanto, a aglomeração de animais é um fator altamente estressante e prejudicial para os mesmos, pois os priva de uma distância individual adequada, impossibilitando movimentos de fuga, agravando 
problemas hierárquicos como a relação entre os indivíduos dominantes e subordinados (Fernandes et al., 2017b).

$\mathrm{Na}$ atualidade, o melhor indicador para determinar a densidade de carga dos compartimentos dos veículos de transporte de animais é através de $\mathrm{m}^{2}$ por animal (González et al., 2012), pois as características dos animais, especialmente os que tem chifres, podem elevar ainda mais o número e o grau de lesões (González et al., 2012). Por isso, a distribuição correta dos animais, associado às densidades, na hora de iniciar a viagem até o abatedouro frigorífico tem que ser estudado como maior determinação. Porém, o tipo de carroceria, varia entre países, de pequena com um só piso, a grande de dois e três andares, existem diferenças consideráveis em resposta ao nível, principalmente quando são transportados animais mais reativos em modelos que não tem subdivisões dentro do espaço de carga disponível. Isso permite que exista uma maior possibilidade de movimentação, causando quedas e lesões durante o transporte em grandes grupos de animais, especialmente se ocorrerem mudanças bruscas de velocidade ou estradas com curvas acentuadas (Gallo et al., 2005).

Aspectos da relação entre fatores e seus impactos:

A literatura mostra que a maioria dos países produtores de carne bovina possuem perdas ocasionadas por contusões, como pode ser observado em alguns trabalhos realizados no México (Miranda-De La Lama et al., 2014), Chile (Gallo et al., 2005; Strappini et al., 2012), Colômbia (Romero et al., 2014), Namíbia (Hoffmand e Luhl, 2012), Itália (Šímová et al., 2016), Austrália (Santurtun e Phillips, 2015), Uruguai (Huertas et al., 2015), Suécia (Bulitta et al., 2015), África do Sul (Chulayo et al., 2016), Gana (Frimpong et al., 2014), Canadá (González et al., 2012), Japão (Ishiwata et al., 2008), Estados Unidos (Youngers et al., 2017) e Brasil (Andrade et al., 2009; Paranhos da Costa et al., 2012; Groff et al., 2016; Mendonça et al., 2017). Essas perdas possuem valores variados, em função do manejo, tipo animal, qualidade dos veículos utilizados no transporte, distâncias e tempos percorridos, sexo dos animais, condições climáticas, entre outros.

$\mathrm{Na}$ Colômbia, Romero et al. (2014), ao avaliarem o efeito do manejo pré-abate sobre as características das contusões nas carcaças de zebuínos, relataram que $61 \%$ das carcaças de bovinos apresentaram pelo menos uma contusão.
Essas contusões na sua maioria foram observadas na tuberosidade isquiática (Tuber isquiadicum) $21,3 \%$, coxal (Tuber coxae) $27 \%$ e no lombo (Longissimus dorsi) 16,6\%, com percentuais de severidade dos hematomas de $80,5 \%$, afetando apenas o tecido subcutâneo e 19,5\% o tecido muscular. Os autores concluíram que as baixas densidades de carga no caminhão e o sexo são fatores que aumentaram a probabilidade de contusões presentes em carcaças de bovinos. Densidades menores que $300 \mathrm{~kg} / \mathrm{m}^{2}$ geram aumento de $1 \%$, bem como machos e fêmeas geram incremento de 1 e $3,7 \%$, respectivamente.

A situação não parece ser diferente no Uruguai, onde Huertas et al. (2015), ao avaliarem 1271 carcaças em 13 abatedouros frigoríficos, verificaram que $60 \%$ apresentam algum tipo de lesão, localizadas em zonas de extremidades $(80,03 \%)$, costelas $(8,5 \%)$, dianteiro $(6,02 \%)$ e lombo $(5,45 \%)$. Com relação à severidade, $71 \%$ das lesões afetaram o tecido subcutâneo e $29 \%$ o tecido muscular, ocorrendo, em média, uma perda de $900 \mathrm{~g}$ de carne por carcaça. Resultados inferiores foram reportados no Chile por Strappini et al. (2010), com incidência de hematomas entre $8,6 \%$ e $20,8 \%$, ao avaliar duas plantas frigoríficas distintas, onde a maioria dos hematomas atingiu apenas o tecido subcutâneo com 19,3\%. No Brasil, Sornas et al. (2017) avaliaram 253.583 carcaças abatidas no abatedouro frigorífico da região metropolitana de Curitiba - PR, no período de janeiro de 2012 até dezembro de 2014, relatando que $10,3 \%$ apresentam pelo menos um tipo de lesão, dos quais $7,2 \%$ localizavam-se na região traseira, $5,4 \%$ na região do vazio e $3,7 \%$ na região da costela. Ao avaliar os fatores relacionados às contusões em carcaças bovinas no Estado do Rio Grande do Sul, Mendonça et al. (2018) descreveram que $54,2 \%$ dos animais avaliados apresentavam pelo menos uma lesão na carcaça, sendo o sexo o fator mais importante dos animais, principalmente as fêmeas, as quais apresentaram $64 \%$ de contusões nas carcaças.

No México, Miranda-De la Lama et al. (2012) constataram que $92 \%$ das carcaças apresentaram algum tipo de lesão, produtos de fatores como o manejo inadequado e transporte com altas densidades. Do total dos hematomas, $14 \%$ localizavam-se no dianteiro, $27 \%$ na costela e no lombo e 59\% no traseiro. Quanto à severidade, $68 \%$ afetaram apenas o tecido subcutâneo, $30 \%$ o tecido muscular e $2 \%$ o tecido ósseo. 
Em Gana, Frimpong et al. (2014) observaram que $82,2 \%$ das carcaças apresentavam hematomas. Com relação à severidade, cerca de $60 \%$ apresentavam contusões leves, enquanto $22 \%$ apresentavam hematomas graves. Os autores destacaram que o manuseio pré-abate tem efeito direto na qualidade e bem-estar animal.

Avaliando a relação entre a prevalência de chifres e a prevalência de hematomas em carcaças de bovinos de corte nos Estados Unidos, Youngers et al. (2017) reportaram que 55,2\% das 4.287 carcaças bovinas apresentaram pelo menos um tipo de contusão. Além disso, a maioria dos hematomas $(61,8 \%)$ ocorreram na linha média dorsal, com $18,6 \%$ e $19,5 \%$ nos lados esquerdo e direito, respectivamente. A prevalência de contusões no terço caudal foi de $21,8 \%$, no centro foi de $60,5 \%$ e na região anterior foi de $17,6 \%$. Segundo os autores a seleção genética para animais sem chifre, poderia influenciar na redução de contusões nas carcaças.

A necessidade de boas práticas de manejo durante $o$ abate realizado no abatedouro frigorifico é muito importante para a cadeia produtiva, devido a que o mercado consumidor incrementa as exigências por produtos de boa qualidade (Barcellos et al., 2012). Hultgren et al. (2014) avaliando as ações relacionadas ao bem-estar animal nas plantas de abate da Suécia, verificaram que $34 \%$ dos animais avaliados estavam com seu bem-estar comprometido, evidenciando a necessidade de treinar melhor os recursos humanos. Dessa maneira, a geração de produtos de melhor qualidade possibilita, tanto aos produtores rurais quanto aos estabelecimentos de abate, a redução de perdas, o maior retorno financeiro para o setor, através da apresentação e distribuição de produtos de melhor qualidade e o atendimento das exigências do consumidor, os quais se preocupam com questões que envolvem a qualidade dos produtos que consomem, como a origem e o bemestar dos animais (Barcellos et al., 2012; Groff et al., 2016; Sornas et al., 2017).

As condições a que são expostos os animais na hora do transporte da fazenda até o abatedouro frigorifico, incrementam os níveis de estresse. Dentre eles podemos mencionar a densidade de carga, a duração da viagem, a qualidade do transporte, bem como os estresses mecânico, acústico, nutricional, social e climático, os quais podem causar mudanças nas variáveis fisiológicas e no comportamento, afetando o bem-estar animal e comprometendo a qualidade do produto final (Gallo, 2005; Schuetze et al., 2017).

Em clima temperado europeu, Warner et al. (1986) evidenciaram maior ocorrência de contusões no inverno, causada pelas baixas temperaturas. Entretanto, Gallo et al. (2000), ao avaliar o efeito do tempo de transporte de novilhos antes do abate em relação às perdas de peso, número de contusões e $\mathrm{pH}$ da carcaça, evidenciaram maior perda de peso em animais transportados na primavera-verão $(8,3 \%)$ em comparação com outono-inverno $(7,2 \%)$. Foi observado maior número de contusões em animais transportados no outono-inverno (73\%), em comparação com primavera-verão (30\%), de igual forma, o número de carcaças com $\mathrm{pH}$ igual ou superior a 5,8 aumentaram com o tempo de transporte mais longo nos animais transportados no primavera-verão com $15 \%$, em comparação com os animais transportado no outono-inverno com $12 \%$.

O temperamento dos animais também influencia na ocorrência de hematomas, principalmente nos processos de carregamento nas propriedades e descarregamento no abatedouro frigorífico. Burdick et al. (2011) manifestaram que o processo de carga e descarga dos bovinos pode ser mais estressante do que o próprio evento de transporte, em função do temperamento animal. Os mesmos autores salientaram que o transporte apenas influencia nas concentrações de cortisol, enquanto que o temperamento influência nas concentrações de cortisol e epinefrina, além da frequência cardíaca. Da mesma forma Lambooij et al. (2012) descreveram que a frequência cardíaca aumenta fortemente durante o carregamento, principalmente por alterações na carga emocional, e diminuiu à medida que o transporte começa. Minka e Ayo (2007), avaliando o efeito do comportamento de carga sobre a incidência de lesões traumáticas em 150 bovinos (Bos indicus) transportados por estrada durante a estação seca quente, evidenciaram que $79 \%$ das carcaças apresentaram lesões (graves e/ou leves), concluindo que o processo realizado no transporte de bovinos durante a estação seca, está associado a fatores de estresse que afetam sua saúde, bem como a produtividade e o valor de mercado. Dessa forma, realizar o transporte dos animais, incluindo procedimentos anteriores e posteriores, de forma adequada reduz a chance de ocorrerem contusões nas carcaças, aumentando a rentabilidade da indústria bovina (Huertas et al., 2010). 
Um veículo adequado para o transporte de animais deve manter o posicionamento adequado dos pontos de carga, além de cumprir as leis e regulamentos que orientam o transporte (Eniolorunda et al., 2009). Apesar de a carroceria dos veículos ser feita de diferentes matérias como madeira, aço e alumínio, existem modelos que tem efeitos negativos, principalmente pela pouca ventilação dentro dos compartimentos, uma vez que a temperatura do ar é um fator ambiental importante que deve ser considerado durante o transporte de bovinos, sendo hoje em dia os veículos longos os que mais dificultam o fluxo de ar, reduzindo a eficiência de ventilação, provocando a inquietação dos animais e aumentando a possibilidade de perda do equilíbrio (Mitchell e Kettlewell, 2008). Nesse sentido, Grandin (1993) manifestou que um bom manejo e práticas adequadas de transportes melhoram a produtividade animal através da redução do estresse e de doenças.

Pesquisas realizadas no Brasil evidenciam o incremento de contusões originadas pelo tipo de veículo utilizado, em função do tempo de viagem. Avaliando o efeito de duas distâncias de transporte (75 a $130 \mathrm{~km}$ e 180 a $250 \mathrm{~km}$ ) e três tipos de caminhão (caminhão boiadeiro, carreta de 1 piso e carreta de dois pisos) sobre o bem-estar e a incidência de contusões em 120 bovinos da raça nelore, Da Silva et al. (2016) observaram maior frequência de contusões $(1,09$ e 1,02) em bovinos transportados pela carreta de dois pisos, de igual forma, foi evidenciado níveis mais elevados de estresse psicológico, caracterizados por altas concentrações de cortisol 8,63 $\mu \mathrm{g} / \mathrm{dL}$ nos animais transportados pela carreta de dois pisos por longas distâncias de 180 a $250 \mathrm{~km}$, demonstrando que esse tipo de caminhão deve ser evitado para realizar transporte por longas distâncias nas condições avaliadas.

Determinando os efeitos de um período de repouso em bovinos transportados por estrada durante 36 horas, em relação às mudanças de peso vivo, ao rendimento de carcaça, à presença de hematomas, às variações de $\mathrm{pH}$ e à coloração da carne pós-morte, Gallo et al. (2001) observaram que o transporte de animais por 36 horas afeta negativamente a qualidade de carne, causam maiores reduções no peso corporal e incrementam o número de hematomas graves.

Os prejuízos econômicos causados por um manejo pré-abate ineficiente, são um reflexo das mudanças necessárias por parte dos produtores, os quais na atualidade são os mais afetados devido à desvalorização de seu produto. Polizel Neto et al. (2015), ao investigar as perdas econômicas decorrentes de lesões de transporte e manejo préabate em 1.021 carcaças de bovinos abatidos no matadouro/frigorífico na região norte de Mato Grosso - MT, Brasil, observaram que $433(42,4 \%)$ apresentavam carcaças com lesões, encontrando perda de $115,76 \mathrm{Kg}$ para o total de animais estudados, estimando que um matadourofrigorífico de porte médio acumula uma perda anual superior a 200 mil reais com lesões em carcaças bovinas decorrentes do transporte.

Mendonça et al. (2016), ao avaliar o grupo genético e presença de chifres em contusões e perdas econômicas em carcaças bovinas, observaram que a maior perda ocorreu no grupo dos zebuínos, totalizando $1,21 \mathrm{Kg} /$ carcaça $(\mathrm{R} \$$ 15,48/animal). A presença de chifres resultou em perda média de $1,13 \mathrm{Kg} /$ carcaça $(\mathrm{R} \$$ 16,11 /carcaça), sendo a região da costela a mais afetada, em comparação aos animais não aspados.

Ao quantificar o impacto econômico da ocorrência de lesões em 490 carcaças de bovinos abatidos no Sudeste do Estado do Pará, Brasil, De Oliveira Melo et al. (2015) observaram que 99\% dos animais avaliados apresentaram ao menos um tipo de lesão, com descarte de $2,33 \mathrm{Kg}$ de carne por animal, sendo a região do quarto traseiro a de maior prevalência de lesões, com prejuízos econômicos, tanto para o produtor $(\mathrm{R} \$ 11,67 /$ animal) como para o abatedouro frigorífico ( $\mathrm{R} \$ 13,41 /$ animal). Da mesma forma, Petroni et al. (2013) avaliando a ocorrência de contusões decorrentes do transporte em 898 carcaças bovinas na Região Centro-Oeste do Estado de São Paulo, Brasil, manifestaram que 98\% dos animais avaliados apresentavam contusões, sendo a região do coxão a mais afetada, com perdas de $117,3 \mathrm{~g}$ por animal, tendo um prejuízo de $\mathrm{R} \$ 0,70$ por animal, somente pelas lesões no coxão.

No Uruguai, Huertas et al. (2015) descreveram que o número médio de bovinos abatidos por ano é de cerca de 2,5 milhões de cabeças, porém quase 2 milhões de toneladas de carne de alta qualidade são desperdiçadas a cada ano, evidenciando uma perda financeira de aproximadamente US\$ 8 bilhões/ano, devido à presença de hematomas, levando em consideração o preço médio da carne em torno de US\$ $4 / \mathrm{Kg}$. 


\section{Considerações Finais}

A presença contusões nas carcaças dos animais constituem em um indicador de problemas de falhas de manejo e bem-estar durante as etapas do pré-abate, bem como de perdas econômicas e prejuízos à totalidade da cadeia produtiva. A implementação de estratégias que permitam diminuir o número de lesões ocasionadas na pecuária de corte, devem contemplar o aumento de capacitações da equipe encarregada do manejo dos animais, assim como o incremento de fiscalizações, as quais garantam que a lei seja cumprida em todo o sistema produtivo, uma vez que a falta das mesmas origina um acrescimento significativo na quantidade de contusões, deteriorando a qualidade da carne e trazendo perdas consideráveis de bemestar aos animais, econômicas aos produtores e aos estabelecimentos de abate, bem como da qualidade do produto aos consumidores.

\section{Referências}

Andrade, E.D.; Silva, R.A.M.S.; Roça, R.D.O. Manejo pré-abate de bovinos de corte no pantanal, Brasil. Archivos de Zootecnia, 58(222): 301-304, 2009.

Andrade, E.N.D.; Silva, R.A.M.S.; Oliveira Roça, R.D.; Silva, L.A.C.D.; Gonçalves, H.C.; Pinheiro, R.S.B. Ocorrência de lesões em carcaças de bovinos de corte no Pantanal em função do transporte. Ciência Rural, 38(7): 1991-1996, 2008.

Anualpec - Anuário da Pecuária Brasileira. 24a ed. Instituto FNP, São Paulo, SP, Brasil. 2017. $288 \mathrm{p}$.

Barcellos, J.O.J.; Abicht, A.D.M.; Brandão, F.S.; Canozzi, M.E.A.; Collares, F.C. Consumer perception of Brazilian traced beef. Revista Brasileira de Zootecnia, 41(3), 771-774, 2012.

Bertoloni, W.; Silva, J.L.D.; Abreu, J.S.D.; Andreolla, D.L. Bem-estar e taxa de hematomas de bovinos transportados em diferentes distâncias e modelos de carroceria no estado do mato grosso-brasil. Revista Brasileira de Saúde e Produção Animal, 13(3): 850-859, 2012.

Bethancourt-Garcia, J.A.; Vaz, R.Z.; Vaz, F.N.; Silva, W.B.; Pascoal, L.L.; Mendonça, F.S.; Vara, C.C.; Nuñez, A.J.C.; Restle, J. Preslaughter factors affecting the incidence of severe bruising in cattle carcasses. Livestock Science, 222: 41-48, 2019.

Bulitta, F.S.; Aradom, S.; Gebresenbet, G. Effect of transport time of up to 12 hours on welfare of cows and bulls. Journal of Service Science and Management, 8: 161-182, 2015.

Burdick, N.C.; Carroll, J.A.; Randel, R.D.; Willard, S.T.; Vann, R.C.; Chase, C.C. Influence of temperament and transportation on physiological and endocrinological parameters in bulls. Livestock Science, 139(3): 213-221, 2011.

Chambers, P.G.; Grandin, T.; Heinz, G.; Srisuvan, T. Guidelines for humane handling, transport and slaughter of livestock. Disponível em: <http://www.fao.org/3/ax6909e.pdf> Acesso em: 24 abr. 2019.

Chulayo, A.Y.; Bradley, G.; Muchenje, V. Effects of transport distance, lairage time and stunning efficiency on cortisol, glucose, hspala and how they relate with meat quality in cattle. Meat Science, 117: 89-96, 2016.

CNT. Confederação Nacional de Transportes, Boletim Estatístico. Disponível em: $<$ http://pesquisarodovias.cnt.org.br/Down loads/Edicoes//2016/Informa\%C3\%A7\%C3 \%B5es\%20para\%20Imprensa/Resumo_Princ ipais_Dados_Pesquisa_CNT_2016_FINAL\% 20(1).pdf/>. Acesso em: 24 abr. 2019.

Da Silva, J.L.; Bertoloni, W.; De Abreu Ribeiro, J.S. Indicadores de estresse e qualidade de carne de bovinos transportados em diferentes tipos de caminhões (tradicional, carreta de um piso e carreta de dois pisos) e diferentes distâncias na região de Cuiabá/MT/Brasil. Archives of Veterinary Science, 21(3): 6876, 2016.

De Oliveira Melo, W.; Santos, E.A.; Abud, L.J.; De Jesus Coelho, G.; Santos, S.C.; Do Rosário Almeida, L.R. Impacto econômico da ocorrência de lesões em carcaças de bovinos abatidos no sudeste do Pará: Influências da presença de traumas e abscessos e da distância entre a fazenda e o abatedouro. Acta Veterinaria Brasilica, 9(3): 243-250, 2015.

Duarte, J.S.; Biazolli, W.; Honorato, C.A. Perdas econômicas devido ao manejo pré-abate: bemestar animal. Comunicação e Mercado/UNIGRAN - Dourados - MS, 3(7): 4-15, 2014.

Eniolorunda, O.O.; Fashina, O.E.; Aro, O.O. Adaptive physiological response to load time stress during transportation of cattle in Nigeria. Archivos de Zootecnia, 58(222), 2009.

Fernandes, T.A.; Costa, P.T.; Farias, G.D.; Vaz, 
R.Z.; Silveira, I.D.B.; Moreira, S.M.; Silveira, R.F. Características comportamentais dos bovinos: Aspectos básicos, processo de aprendizagem e fatores que as afetam. Revista Eletrónica de Veterinaria, 18(9): 1-16, $2017 \mathrm{a}$.

Fernandes, T.A.; Costa, P.T.; Farias, G.D.; Vaz, R.Z.; Silveira, I.D.B.; Moreira, S.M.; Silveira, R. F. Características comportamentais dos bovinos: Influências da domesticação e da interação homem-animal. Revista Eletrónica de Veterinaria, 18(12): 1-29, 2017b.

Frimpong, S.; Gebresenbet, G.; Bobobee, E.; Aklaku, E.D.; Hamdu, I. Effect of transportation and pre-slaughter handling on welfare and meat quality of cattle: case study of Kumasi abattoir, Ghana. Veterinary Sciences, 1(3): 174-191, 2014.

Gallo, C.; Néstor, T. Bienestar animal y calidad de carne durante los manejos previos al faenamiento en bovinos. Revista electrónica de Veterinaria, 9(10): 1695-7004, 2008.

Gallo, C.; Warriss, P.; Knowles, T.; Negrón, R.; Valdés, A.; Mencarini, I. Densidades de cargas utilizadas para el transporte de bovinos destinados a matadero en Chile. Archivos de Medicina Veterinaria, 37(2): 155-159, 2005.

Gallo, C.; Espinoza, M.A.; Gasic, J. Efectos del transporte por camión durante 36 horas con y sin período de descanso sobre el peso vivo y algunos aspectos de calidad de carne en bovinos. Archivos de Medicina Veterinaria, 33(1): 43-53, 2001.

Gallo, S.C.; Perez, V.S.; Sanhueza, V.C.; Gasic, Y.J. Efectos del tiempo de transporte de novillos previo al faenamiento sobre el comportamiento, las pérdidas de peso y algunas características de la canal. Archivos de Medicina Veterinaria, 32(2): 157-170, 2000.

González, L.A.; Schwartzkopf-Genswein, K.S.; Bryan, M.; Silasi, R.; Brown, F. Space allowance during commercial long distance transport of cattle in North America. Journal of Animal Science, 90(10): 3618-3629, 2012.

Grandin, T. Management commitment to incremental improvements greatly improves livestock handling. Meat Focus. 1993. 450-453p.

Groff, A.M.; Bassani, C.A.; Pianho, C.R. Causas de condenação total de carcaças bovinas em um frigorífico do estado do paraná. Relato de caso. Revista Brasileira de Higiene e
Sanidade Animal, 10(4): 730-741, 2016.

Hoffman, L.C.; Lühl, J. Causes of cattle bruising during handling and transport in Namibia.

Meat Science, 92(2): 115-124, 2012.

Huertas, S.M.; Eerdenburg, F.; Gil, A.; Piaggio, J. Prevalence of carcass bruises as an indicator of welfare in beef cattle and the relation to the economic impact. Veterinary Medicine and Science, 1(1): 9-15, 2015.

Huertas, S.M.; Gil, A.D.; Piaggio, J.M.; Van Eerdenburg, F.J.C.M. Transportation of beef cattle to slaughterhouses and how this relates to animal welfare and carcase bruising in an extensive production system. Animal Welfare, 19(3): 281-285, 2010.

Hultgren, J.; Wiberg, S.; Berg, C.; Cvek, K.; Kolstrup, C.L. Cattle behaviours and stockperson actions related to impaired animal welfare at Swedish slaughter plants. Applied Animal Behaviour Science, 152: 23-37, 2014.

Ishiwata, T.; Uetake, K.; Eguchi, Y.; Tanaka, T. Steer stress levels during long distance transport throughout the year in japan. Animal Science Journal, 79(4): 510-517, 2008.

Lambooij, E.; Reimert, H.G.M.; Van der Werf, J.T.N.; Hindle, V.A. Compartment height in cattle transport vehicles. Wageningen UR Livestock Research, 148(1-2): 87-94, 2012.

Ludtke, C.B.; Ciocca, J.R.P.; Dandin, T.; Barbalho, P.C.; Vilela, J.A.; Ferrarini, C. Abate humanitário de bovinos. Rio de Janeiro: WSPA, 2012. 147p.

Marques, R.S.; Cooke, R.F.; Francisco, C.L.; Bohnert, D.W. Effects of twenty-four hour transport or twenty-four hour feed and water deprivation on physiologic and performance responses of feeder cattle. Journal of Animal Science, 90(13): 5040-5046, 2012.

Mendonça, F.S.; Vaz, R.Z.; Cardoso, F.F.; Restle, J.; Vaz, F.N.; Pascoal, L.L.; Reimann, F.A.; Boligon, A.A. Pre-slaughtering factors related to bruises on cattle carcasses. Animal Production Science, 58: 385, 2018.

Mendonça, F.S.; Vaz, R.Z.; Vaz, F.N.; Restle, J.; Gonçalves, G.B.; Vara, C.C.D. Características raciais e de carcaça nas perdas por contusões e no $\mathrm{pH}$ final da carne de bovinos machos castrados e fêmeas de descarte. Ciência Animal Brasileira, 18:1-10, 2017.

Mendonça, F.S.; Vaz, R.Z.; Silveira, W.L.; Restle, J. Pascoal, L.L.; Bitencourt, M.V.; Duarte 
G.F. Genetic group and horns presence in bruises and economic losses in cattle carcasses. Semina: Ciências Agrárias, 37(6): 42654273, 2016.

Minka, N.S.; Ayo, J.O. Effects of loading behaviour and road transport stress on traumatic injuries in cattle transported by road during the hot-dry season. Livestock Science, 107(1): 91-95, 2007.

Miranda-De La Lama, G.C.; Villarroel, M.; María, G.A. Livestock transport from the perspective of the pre-slaughter logistic chain: A Review. Meat Science. 98(1): 9-20, 2014.

Miranda-De La Lama, G.C.; Leyva, I.G.; BarrerasSerrano, A.; Pérez-Linares, C.; SánchezLópez, E.; María, G.A. Assessment of cattle welfare at a commercial slaughter plant in the northwest of Mexico. Tropical Animal Health and Production, 44(3): 497-504, 2012.

Mitchell, M.A.; Kettlewell, P.J. Engineering and design of vehicles for long distance road transport of livestock (ruminants, pigs and poultry). Veterinária Italiana, 44(1): 201213, 2008.

Mitchell, L. Impact of consumer demand for animal welfare on global trade. Changing Structure of Global Food Consumption and Trade, 80: 83-85, 2001.

Paranhos da Costa, M.J.P.; Huertas, S.M.; Gallo, C.; Dalla Costa, O.A. Strategies to promote farm animal welfare in Latin America and their effects on carcass and meat quality traits. Meat Science, 92(3): 221-226, 2012.

Pereira de Abreu, D.A.; Cruz, J.M.; Losada, P.P. Active and intelligent packaging for the food industry. Food Reviews International, 28(2): 146-187, 2012.

Petroni, R.; Burger, K.P.; Gonçalvez, P.O.; Rossi, G.A.M.; Vidal-Martins, A.M.C.; Aguilar, C.E.G. Ocorrência de contusões em carcaças bovinas em frigorífico. Revista Brasileira de Saúde e Produção Animal, 14(3): 478-484, 2013.

Polizel Neto, A.; Zanco, N.; Lolatto, D.C.; Moreira, O.S.; Dromboski, T. Perdas econômicas ocasionadas por lesões em carcaças de bovinos abatidos em matadourofrigorífico do norte de Mato Grosso. Pesquisa Veterinária Brasileira, 35(4): 324-328, 2015.
Romero, M.H.; Uribe-Velásquez, L.F.; Sánchez, J.A. Effect of pre-slaughter handling on the characteristics of bruises in beef carcasses into two Colombian slaughter plants. Revista Colombiana de Ciencias Pecuarias, 8(1): 116, 2014.

Santurtun, E.; Phillips, C.J. The impact of vehicle motion during transport on animal welfare. Research in Veterinary Science, 100: 303308, 2015.

Schuetze, S.J.; Schwandt, E.F.; Maghirang, R.G.; Thomson, D.U. Transportation of commercial finished cattle and animal welfare considerations. The Professional Animal Scientist, 33(5): 509-519, 2017.

Šímová, V.; Večerek, V.; Passantino, A.; Voslářová, E. Pre-transport factors affecting the welfare of cattle during road transport for slaughter-A Review. Acta Veterinaria Brunensis, 85(3): 303-318, 2016.

Sornas, A.; Rossi Junior, P.; Moizes, F. Losses occasioned by injuries in bovine carcass and its economic reflection in the state of Paraná. Archives of Veterinary Science, 21(3): 119130, 2017.

Strappini, A.C.; Frankena, K.; Metz, J.H.M.; Gallo, C.; Kemp, B. Characteristics of bruises in carcasses of cows sourced from farms or from livestock markets. Animal, 6(3): 502-509, 2012.

Strappini, A.C.; Frankena, K.; Metz, J.H.M.; Gallo, B.; Kemp, B. Prevalence and risk factors for bruises in Chilean bovine carcasses. Meat Science, 86(3): 859-864, 2010.

Tucker, C.B.; Rogers, A.R.; Schütz, K.E. Effect of solar radiation on dairy cattle behaviour, use of shade and body temperature in a pasturebased system. Applied Animal Behaviour Science, 109(2-4), 141-154, 2008.

Warner, R.D.; Eldridge, G.A.; Barnett, J.L.; Cahill, D.J.; Halpin, C.G. The effects of fasting and cold stress on dark cutting and bruising in cattle. Proceedings of the Australian Society of Animal Production, 16:383-386, 1986.

Youngers, M.E.; Thomson, D.U.; Schwandt, E.F.; Simroth, J.C.; Bartle, S.J.; Siemens, M.G. Case study: Prevalence of horns and bruising in feedlot cattle at slaughter. The Professional Animal Scientist, 33(1): 135139, 2017. 